\title{
Sprachliche Persuasionsmittel der rechtspopulistischen Propaganda gestern und heute
}

\author{
Vincenzo Gannuscio (Modena)
}

\begin{abstract}
Persuasion is the central element of political propaganda of all times. From a historical point of view, it is a fact that, especially in conflict situations, the hegemonic actors adopt specific techniques and language choices for purposes of propaganda. The question of interest is in what ways and to what extent they are used in a targeted manner in order to influence different types of low- and middle-class audiences. A well-documented example is the persuasive use of language in Nazi propaganda before and during World War II. In this context, our question is whether past and present-day propagandistic and electoral campaigns make recourse to the same linguistic devices and strategies in order to persuade their intended audience. To this purpose, we shall concentrate on Hitler's propaganda by primarily drawing on Victor Klemperer's (1933-1945) diaries and compare his linguistic observations to a corpus of campaigning speeches and programs by the German right-wing populist party AfD (Alternative für Deutschland).
\end{abstract}

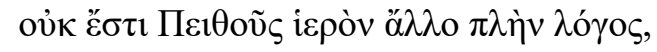

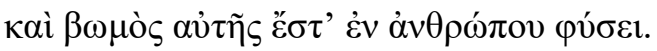

(Euripides, Antigone - Fragment 170) ${ }^{1}$

\section{$1 \quad$ Prämissen}

Nicht nur in Deutschland haben jüngste Wahlergebnisse durch ein vermehrtes Wiederaufkommen rechtspopulistischer Bewegungen das etablierte politische System erschüttert. Denn vermeintlich neue Parteien entfalten derzeit eine mitreißende und unerwartete propagandistische Persuasionskraft, die erfahrene Akteure der demokratischen Auseinandersetzung als überholt und anachronistisch wirken lässt. Nicht zufällig ruft die Sprache, die die Rechtspopulisten anwenden, dunkle Zeiten in Erinnerung, die die Geschichte vorbehaltlos verurteilt hat.

Ziel vorliegender Darlegungen ist es zu zeigen, wie die politische Propaganda sprachliche Mittel einsetzt und in einem bestimmten historischen Kontext eingesetzt hat und wie sie gelegentlich das Wortgut einer mehr oder weniger starken Verschiebung des semantischen Wertes unterzieht, um das eigene persuasorische Vorhaben umzusetzen.

In Anlehnung an Perelman/Olbrechts-Tytecas Unterscheidung zwischen Persuasion und Überzeugung wird davon ausgegangen, dass Persuasion und nicht Überzeugung ein zentrales

\footnotetext{
1 „Die Persuasion hat keinen anderen Tempel als das Wort und ihr Altar ist in der Natur des Menschen.“ (Collard/Cropp 2008: 166).
} 
Anliegen der Propaganda ist, denn dieser kommt es vielmehr auf das effektive Erreichen des erwünschten Resultats an als auf das Erzielen rationaler Unterstützung und Adhäsion, die eher durch Überzeugung zu gewinnen ist:

Pour qui se préoccupe du résultat, persuader est plus que convaincre, la conviction n'étant que le premier stade qui mène à l'action. [...] Par contre, pour qui est préoccupé du caractère rationnel de l'adhésion, convaincre est plus que persuader. ${ }^{2}$

(Perelman/Olbrechts-Tyteca 1970: 35)

Weiterer Ansatzpunkt ist die Einsicht, dass Sprache ein unabdingbares Instrument der propagandistischen Persuasion ist. Schon als Papst Gregor XV im Jahr 1622 die Sancta congregatio de propaganda fide gründete, war das Wort Gottes, und somit das sprachliche Mittel, das grundlegende Instrument der päpstlichen Institution, deren Namen (Propaganda) man dann zur Bezeichnung des säkularen, politischen Persuasionsprozesses übernahm. Interessant zu bemerken ist übrigens, dass der Begriff „Propaganda“ schon recht früh, sogar noch vor seiner Säkularisierung und Politisierung, mit Verschwörungstheorien assoziiert wurde und somit die heutige abwertende Färbung annahm (cf. Schmitz-Berning 1998: 475).

Sprachhistorisch kann man belegen, wie sich die hegemonischen Akteure besonders in Konfliktsituationen des propagandistischen Mittels bemächtigen und wie sie es gezielt einsetzen, um breite Gesellschaftsschichten zu beeinflussen. Demgemäß kann vorliegende Recherche nicht umhin, am persuasiven Propaganda-Repertoire des nationalsozialistischen Regimes anzusetzen, wozu eine reich dokumentierte wissenschaftliche Literatur vorhanden ist. Ausgehend von Victor Klemperers (1933-1945) Tagebüchern und von den sprachlichen Beobachtungen, die in seinem 1947 erschienenen Band LTI - Lingua Tertii Imperii enthalten sind, sollen zunächst die linguistischen Persuasionsmittel der NSDAP-Propaganda aus der Sicht des zum inneren Exil verurteilten Philologen herauskristallisiert werden. Darauf basierend soll weiter festgestellt werden, ob und inwiefern in den heutigen Wahlkampfreden und Programmen rechtspopulistischer Parteien, hier spezifisch der Alternative für Deutschland (AfD), die gleichen sprachlichen Mittel und Strategien vorkommen, was die Schlussfolgerung erlaubte, in der aktuellen Kommunikation der Politiker fließe, um es salopp zu sagen, nichts anderes als ,alter Wein in neuen Schläuchen“.

\footnotetext{
2 Die Übersetzung ins Deutsche des Vokabulars des Traité de l'argumentation bereitet nicht wenig Schwierigkeiten. Lässt sich convaincre gut mit überzeugen wiedergegeben, ist die Übersetzung von persuader etwas heikler. In offiziellen Übersetzungen des Traité de l'argumentation wird das Verb persuader mit überreden wiedergegeben, dies sollte jedoch ausgehend von folgenden Wörterbuchdefinitionen kritisch hinterfragt werden:

„überreden: durch [eindringliches $\mathrm{Zu}$ ]reden dazu bringen, dass jemand etwas tut, was er ursprünglich nicht wollte“. (Duden online s. v. überreden);

,persuader: amener (qqn) à croire, à penser, à vouloir, à faire (qqch.), par une adhésion complète (sentimentale autant qu'intellectuelle). [...] Exemple: Il m'a persuadé de la sincérité de ses intentions. (Le Nouveau Petit Robert, s. v. persuader).

Das dt. überreden ist handlungsbezogen und geht davon aus, dass der Adressat der Argumentation die gewünschte Handlung ursprünglich nicht ausführen wollte. Diese Bedeutungskomponente ist in persuader weniger explizit. Persuader kann laut Wörterbuchdefinition auch nicht handlungsbezogen sein (cf. das zitierte Beispiel), auch wenn Perelman und Olbrechts-Tyteca das Verb ausschließlich in diesem Sinne verstanden wissen wollen.
} 


\section{$1.1 \quad$ Victor Klemperer (1881-1960)}

Im deutschen Kulturraum wurde der Name Klemperers lange Zeit fast ausschließlich mit der weitaus berühmteren Figur des Dirigenten Otto Klemperer verbunden. Dessen Cousin, Victor Klemperer, war bis Ende des 20. Jahrhunderts nur einem beschränkten Publikum im Bereich der Romanistik und der Sprachgeschichte bekannt. ${ }^{3}$ Der Philologe und Romanist Victor Klemperer war das achte Kind eines Rabbiners der jüdischen Reformgemeinde Berlins. Das Vorbild des Vaters, der dank einer moderneren Auslegung der talmudischen Gesetze keineswegs eine orthodoxe oder gar selbstverzehrende Lebensart führte (Jacobs 2010: 7-9), trug dazu bei, dass Klemperers Verbundenheit mit dem Judentum nicht besonders ausgeprägt war und dass er schon recht früh zur protestantischen Konfession konvertierte.

Klemperer schrieb in sein Tagebuch „Ich habe mich wahrhaftig immer als Deutscher gefühlt” (Klemperer 1999a: 15), und in der Tat war er ein überzeugter Patriot, was auch dadurch bezeugt wird, dass er sich im Ersten Weltkrieg freiwillig zur Armee meldete. Nach dem Krieg erlangte er an der Technischen Universität Dresden einen Lehrstuhl für Romanistik, den er bis 1935 innehatte; dann wurde er durch die Nazis seines Amtes enthoben. Es begann nun für ihn eine sehr schwierige Zeit: Aus seinem Haus vertrieben und als Zwangsarbeiter verpflichtet, blieb ihm nur dank der Ehe mit einer arischen Frau das Schicksal vieler Juden erspart.

Die ihm aufgezwungene Inaktivität nutzte er, um in seinen Tagebüchern Eindrücke und Gedanken aus dem Alltag festzuhalten. Diese Tagebuch-Aufzeichnungen blieben glücklicherweise erhalten und stießen in den 90er Jahren, nach ihrer postumen Veröffentlichung, auf ein breites Interesse. Victor Klemperer musste sich nach der nationalsozialistischen Machtergreifung mit der schwer zu akzeptierenden Tatsache konfrontieren, dass seine Kultur, seine Nation und seine Muttersprache ihm Leiden und Misere aufzwangen:

Ich glaube nicht mehr an Völkerpsychologie. Alles, was ich für undeutsch gehalten habe, Brutalität, Ungerechtigkeit, Heuchelei, Massensuggestion bis zur Besoffenheit, alles das floriert hier.

(Klemperer 1999a: 15)

Für den Sprachhistoriker ist neben den Tagebüchern Victor Klemperers LTI - Lingua Tertii Imperii (1947) von besonderer Bedeutung. In diesem Band setzt sich der Dresdner Philologe ausgiebig mit der Sprache im Dritten Reich auseinander. Sich der Komplexität des Phänomens der Nazi-Sprache bewusst, bezeichnet Klemperer sein Werk als „Notizbuch“ und kommentiert einleitend seine Arbeit wie folgt:

Denn es wird sehr viel Facharbeit auf verschiedenen Gebieten zu leisten sein, Germanisten und Romanisten, Anglisten und Slawisten [sic], Historiker und Nationalökonomen, Juristen und Theologen, Techniker und Naturwissenschaftler werden in Exkursen und ganzen Dissertationen sehr viele Einzelprobleme zu lösen haben, ehe ein mutiger und umfassender Kopf es wagen darf die Lingua Tertii Imperii in ihrer Gesamtheit, der allerarmseligsten und aller reichhaltigsten Gesamtheit, darzustellen.

(Klemperer 1996: 22)

\footnotetext{
${ }^{3}$ Dies änderte sich mit der Veröffentlichung seiner Tagebücher und infolge einer 1999 von der ARD ihm gewidmeten Fernsehserie. Die 13-teilige Serie Klemperer - Ein Leben in Deutschland (Regie: Kai Wessel/Andreas Kleinert, Drehbuch: Peter Steinbach) hatte großen Erfolg und erweckte neues Interesse an den Schriften Klemperers.
} 
Der Band versammelt Klemperers Untersuchungen zum Zusammenhang zwischen Sprache und Ideologie des Nationalsozialismus und darf zweifellos als einer der wichtigsten Beiträge zur Darstellung der ideologischen Mechanismen des Nazi-Regimes sowie des gezielten und effizienten Einsatzes der Massenkommunikationsmedien zu persuasorischen Zwecken angesehen werden. Wie u. a. Nicoletta Cavazza (2006) in ihrer gesellschaftspsychologischen Studie zur Persuasion hervorhebt, verhelfen Massenkommunikationsmedien breiteren Gesellschaftsschichten zu einer Meinungs- und Willensbildung, die sonst mangels Information ausbliebe, und gewinnen folglich politische und soziale Relevanz, insofern als sie die Adressaten in den Stand setzen, in jenen Bereichen Entscheidungen zu treffen, für die sie keine direkte Erfahrung bzw. Kenntnis aus erster Hand haben. Durch die Einflussnahme auf die öffentliche Meinungsbildung konkretisiert sich also die Möglichkeit, die Entscheidungen der Masse in eine bestimmte Richtung zu lenken und somit das Leitprinzip des Pluralismus zu umgehen:

Luogo privilegiato della persuasione sono i mezzi di comunicazione di massa, che permettono al pubblico di assumere informazioni, formulare giudizi e decisioni in ambiti per i quali non ha conoscenze di prima mano. In questa dipendenza si insinua la possibilità di esercitare una certa influenza sulla direzione che giudizi e decisioni prendono. Lo studio degli effetti persuasivi che i mezzi di comunicazione di massa possono avere nell'ambito dei giudizi e delle decisioni politiche assume una forte rilevanza sociale.

(Cavazza 2006: 155)

Die NSDAP bemächtigte sich einerseits meisterhaft dieses Potentials der Massenkommunikationsmedien (cf. diesbezüglich u. a. Ehlich 1989: 20) und vollführte andererseits einen semantischen Verwandlungsprozess des alltäglichen Wortschatzes:

Die propagandistische Stärke des NS-Sprachgebrauchs bestand ja gerade in der semantischen Engführung der vorfindlichen Begrifflichkeiten, die dem politisch weniger Interessierten und Informierten ein einfaches, leicht zu handhabendes Instrumentarium bot, komplizierte Sachverhalte zu begreifen, nicht zuletzt mittels Reduzierung auf scheinbar eindeutige Feindbilder und auf scheinbar unproblematische Lebensziele.

(Schlosser 2013: 11)

Laut diesen Beschreibungsansätzen kommt dem Publikum eine ausschließlich passive Rolle zu, als würde sich in dem Zuhörer ein akritisches In-sich-hineinfließen-Lassen der sprachlichen Persuasionselemente abspielen. Diese Passivität bezeugt auch Victor Klemperer:

Was war das stärkste Propagandamittel der Hitlerei? Waren es Hitlers und Goebbels' Einzelreden, ihre Aufführungen zu dem und jenem Gegenstand, ihre Hetze gegen das Judentum, gegen den Bolschewismus? Fraglos nicht, denn vieles blieb von der Masse unverstanden oder langweilte sie in seinen ewigen Wiederholungen. Wie oft in Gasthäusern, [...] wie oft später in der Fabrik

während der Luftwache, [...] wie oft habe ich die Spielkarten auf den Tisch klatschen und laute Gespräche über Fleisch- und Tabakrationen und über das Kino führen hören, während der Führer oder einer seiner Paladine langatmig sprachen, und nachher hieß es in den Zeitungen, das ganze Volk habe ihnen gelauscht.

(Klemperer 1996: 24)

Klemperer ist davon überzeugt, dass die effektive Persuasion der Masse in der Nazizeit nicht durch Reden, Artikel, Flugblätter oder Plakate erzielt wurde, da diese mit bewusstem Denken aufgenommen werden mussten. In Anlehnung an das berühmte Schiller-Distichon von der 
„gebildeten Sprache, die für uns dichtet und denkt" ${ }^{\text {" }}$ glaubte Klemperer an eine weitgehende Steuerung von Gefühl und Verstand durch Worte. Er war davon überzeugt, dass sich das Regime durch millionenfache Wiederholung von Parolen und Redewendungen, die mechanisch und unbewusst übernommen wurden, durchsetzte. Um diesen Prozess zu veranschaulichen, griff er auf folgenden, schon oft zitierten Vergleich zurück:

Worte können sein wie winzige Arsendosen: sie werden unbemerkt verschluckt, sie scheinen keine Wirkung zu tun, und nach einiger Zeit ist die Giftwirkung doch da.

(Klemperer 1996: 25)

Spätere Studien haben laut Göttert (2010: 315) bewiesen, dass Klemperer dabei von einer falschen Voraussetzung ausging. Als Philologe lebenslang mit nichts anderem als Sprache beschäftigt, maß er den Worten eine übergroße Macht bei. Ein halbes Jahrhundert sprachwissenschaftlicher Forschung sollte allerdings zeigen, dass Verführungs- und Lenkungsmacht einzelner Worte, Parolen und Redewendungen begrenzt ist, und dass die Verführung vielmehr von einem komplexeren Zusammenspiel aller konstitutiven Elemente des kommunikativen Sprachhandelns ausgeht. Und doch sind Klemperers Notizen in LTI und in seinen Tagebüchern sehr nützlich, nicht zuletzt deswegen, weil sie sich als idealer Leitfaden für eine Gegenüberstellung der Sprache der nationalsozialistischen Propaganda und derjenigen der neuen rechtspopulistischen Alternative für Deutschland anbieten.

\subsection{AfD: Alternative für Deutschland}

Die 2013 in Berlin als Reaktion auf die Euro-Rettungspolitik gegründete Partei Alternative für Deutschland (AfD) hat in kürzester Zeit eine Resonanz erlangt, die dank der nunmehr fast täglichen Schlagzeilen weit über die BRD-Landesgrenzen hinausreicht. Schon ein Jahr nach ihrer Gründung errang die AfD bei der Europawahl überregionale Mandate und zog gleich danach auch in verschiedene Länderparlamente ein. Heute handelt es sich dabei um eine

Partei die das nachkriegsdeutsche Parteiengefüge verändert hat, die - aufgrund ihrer Positionen die z. T. konservativ-national(istisch), z. T. als fremdenfeindlich, z. T. als rassistische, z. T. als verfassungswidrig bewertet werden - in hohem Maß umstritten ist.

(Kämper 2017: 2f.)

Die Wahlergebnisse der letzten Jahre zeigen (cf. Zicht/Cantow 2017), dass der Konsens der Partei einen stark steigenden Trend registriert hat, bis hin zu den Wahlen des Jahres 2016, bei denen die AfD in Mecklenburg-Vorpommern und Sachsen-Anhalt sogar die 20\%-Grenze überschritten hat. Diese Daten rechtfertigen im Vergleich zu denen, die die nunmehr etablierte Rechtspartei NPD in ihrem bisherigen politischen Leben erzielt hat, das Vorhaben, die Sprache der AfD zu einem Untersuchungsgegenstand zu machen, denn zumindest bis dato hat die AfD eine unbestreitbar persuasorische Kraft besessen.

Die AfD wurde von Anfang an rechts verortet und als populistisch eingestuft. Als signifikanter Rechtsruck wurde der Führungswechsel im Juli 2015 gedeutet, als Frauke Petry und Jörg Meuthen zu Parteivorsitzenden gewählt wurden. Vor allem Frauke Petry und die stellvertretende Bundesvorsitzende Beatrix von Storch sind immer wieder durch ihre Meinungsäuße-

\footnotetext{
${ }^{4}$ Dilettant - Weil ein Vers dir gelingt in einer gebildeten Sprache, die für dich dichtet und denkt, glaubst du schon Dichter zu seyn? (Schiller 2013: 28).
} 
rungen ins Kreuzfeuer der Kritik geraten. So sorgten sie Anfang 2016 für einen medialen Aufschrei, als beide behaupteten, es sei gerechtfertigt, auf Flüchtlinge, sogar auf Kinder, zu schießen (cf. Tomik 2016). Als die Entrüstung überhandnahm, dementierte allerdings Frau von Storch ${ }^{5}$ und meinte, es sei nicht gerechtfertigt, auf Kinder zu schießen, wohl aber auf Frauen, denn diese seien ,„[...] anders als Kinder verständig.“(Ott 2016). Wie von Zeit Online dokumentiert, ist mittlerweile diese Sequenz von Provozieren, Relativieren und dann Dementieren zu einer regelrechten Methode geworden, die der AfD zur konstanten medialen Präsenz verhilft (cf. Steffen 2016).

\subsection{Definition von Rechtspopulismus}

In der politologischen und politolinguistischen Literatur lassen sich grundsätzlich drei definitorische Konstanten erkennen, die sowohl dem Links- als auch dem Rechtspopulismus gemeinsam sind (cf. u. a. Stegemann 2017, 7; Scharloth 2016; Wodak 2015, 25-26; Lewandowsky 2014, 20-21; Cedroni 2014, 40-42):

- Tabubrüche und Skandalisierung: Populistische Parteien stellen die festgelegten Spielregeln in Frage, indem sie Themen aufgreifen, die von den etablierten politischen Mitstreitern generell tabuisiert werden. Im Diskurs mit den politischen Gegnern ersetzt die negative Darstellung durch hyperbolische und oft metaphorische verbale Schmähungen eine kritische und rationale Argumentation.

- Rekurs auf das Volk: Schon der Wortstamm des Begriffs Populismus weist darauf hin, dass im Fokus der Interessen der populistischen Akteure das Volk steht. Populisten verstehen sich dementsprechend als Verteidiger jener sozialen Schichten, die durch das politische Establishment benachteiligt werden. Zu betonen ist dabei, dass der Begriff „Volk“ vornehmlich nicht im Sinne des Staatsvolkes gebraucht, sondern als kulturell und ethnisch homogene Abstammungsgemeinschaft verstanden wird.

- Frontstellung gegen die gesellschaftlichen Eliten: Der Populismus kann als ein auf Abgrenzung basierendes politisches Modell angesehen werden, das seine Wir-Identität im Kontrast zu dem konstruiert, was es zu bekämpfen versucht. Diese Wir/Ihr-Schemata ordnen sich sowohl auf eine vertikale, als auch auf eine horizontale Achse, denn die Freund/Feind-Abgrenzung profiliert sich nicht nur als Frontstellung gegen politisches Establishment, gesellschaftliche Eliten, Banken, Trusts und Kartelle, sondern auch gegen kulturelle, ethnische, religiöse und gegebenenfalls sexuelle „Außenseiter“.

Diese charakterisierenden und konnotativen Konstanten sollen im Folgenden als Analyseraster dienen. Fokussiert werden also: 1) Tabubrüche und Skandalisierung, 2) Rekurs auf das Volk und 3) Frontstellung gegen die gesellschaftlichen Eliten.

\subsection{Zusammensetzung des Analysekorpus}

Datengrundlage vorliegender Analyse ist ein ad hoc zusammengestelltes Korpus aus Wahlprogrammen und transkribierten Audio- und Videodateien. ${ }^{6}$ Letztere wurden in der Annahme

\footnotetext{
${ }^{5}$ Stoff für die politische Satire erbrachte Beatrix von Storchs Äußerung „Mir ist die Maus ausgerutscht!“, mit der sie rechtfertigte, warum sie in einem Online-Forum mit einem lapidaren ,ja“ auf die direkte Frage, ob man auch auf Kinder schießen solle, geantwortet hatte (Matthies 2016).

${ }^{6}$ Zur Transkription wurde die von der Universität Hamburg zur Verfügung gestellte Software EXMARaLDA eingesetzt (http://exmaralda.org [24.06.2019]).
} 
aufgenommen, dass in diesen, anders als in Printtexten, die reflektiert und von einer mehr oder weniger großen Parteiöffentlichkeit approbiert sind (cf. Kämper 2017: 2), ein freieres und unkontrollierteres Sprechen vorkommt, wodurch die Grundeinstellung der Partei ungefiltert anschaulich wird. Eine erste grobe Bestandsaufnahme hat in der Tat auch gezeigt, dass gerade in diesen Texten vermehrt lexikalische Persuasionsmittel und -strategien des politischen Populismus vorzufinden sind. Das AfD-Analysekorpus setzt sich also im Spezifischen folgendermaßen

zusammen:

- AfD Grundsatzprogramm (AfD 2016),

- Entwurf des AfD-Bundestagswahlprogramms (AfD 2017),

- Landtagswahlprogramme (AfD Baden-Württemberg 2016, AfD Berlin 2016, AfD Rheinland-Pfalz 2016, AfD Sachsen 2014, AfD Sachsen-Anhalt 2016),

- Transkripte öffentlicher Reden 7 .

Als Vergleichskorpus dienen analoge Texte anderer Akteure der bundespolitischen Debatte.

\begin{tabular}{|l|c|c|}
\hline & AfD-Korpus & Vergleichskorpus \\
\hline word tokens & 126.616 & 272.513 \\
\hline word types & 18.696 & 26.558 \\
\hline
\end{tabular}

Tabelle 1: Zusammensetzung des Analyse- und Vergleichskorpus 8

\section{Die Sprache der AfD}

\subsection{Tabubrüche und Skandalisierung}

Dass das Auftreten der AfD oft mit Tabubrüchen und Skandalisierung verbunden ist, zeigt schon eine oberflächliche Bestandsaufnahme der Presse. ${ }^{9}$ Wie in einer Studie von Raffaella Petrilli erläutert wird, ist dies aber durchaus kein Einzelfall im zeitgenössischen politischen Panorama Europas. In den letzten Jahren hat man nicht selten ähnliche Schlagzeilen in der französischen Presse in Bezug auf Jean-Marie Le Pen und dessen Tochter Marine lesen können, und auch Italien sind sie nicht erspart geblieben, wenn auch wohl weniger extremistisch ausgerichtet. Am Beispiel der Sprache Beppe Grillos beschreibt Petrilli, wie die politische Sprache populistischer Parteien immer mehr auf Provokation, verbale Aggressivität und Schimpfwörter zurückgreift, um die politischen Gegner mit einem fast theatralischen Gestus zu delegitimieren.

Il discorso politico si è man mano arricchito di espressioni colorite, di metafore goliardiche che mirano a mettere in ridicolo gli avversari, come nel caso di Grillo. [...] La caratteristica del linguaggio di Grillo non risiede tanto nel turpiloquio e nel registro popolaresco, quanto nel fatto

\footnotetext{
${ }^{7}$ Es handelt sich dabei um Reden der ehemaligen Parteivorsitzenden Frauke Petry (2015), der EUParlamentarierin Beatrix von Storch (2015), des Thüringer AfD-Landesvorsitzender Björn Höcke (2016a, 2016b) und der zwei Repräsentanten der Jungen Alternative Markus Frohnmaier (2015) und Sven Werner Tritschler (2016).

8 Die Korpusanalyse wurde mit Sketch Engine (www.sketchengine.co.uk/ [24.06.2019]) durchgeführt.

${ }^{9} \mathrm{Im}$ Gegensatz zu den in den folgenden Absätzen beschriebenen Aspekten lassen sich bezüglich der Tabubrüche und Skandalisierungen ausschließlich qualitative Analysen durchführen.
} 
che l'aggressività verbale sia la norma, nei comizi come nei blog, e sia usata per delegittimare gli avversari politici, secondo il metodo tipico della propaganda. Con Grillo non solo il turpiloquio, ma tutte le forme di aggressività verbale, dalla parolaccia all'insulto, sono entrate sistematicamente nel linguaggio politico, alzandone i toni e abbassandone il livello, soprattutto distogliendo l'attenzione dai contenuti che lo stesso Grillo propone e dalla ripetitività delle sue teorie, espresse con una metodologia assimilabile a quella degli spettacoli teatrali.

(Petrilli 2015: 122f)

Eine von Joachim Scharloth durchgeführte Analyse eines schriftlichen Korpus aus Pressemitteilungen und Wahlprogrammen der Landtagswahlen 2016 in Rheinland-Pfalz bestätigt, dass die AfD nicht an Skandalisierung spart. Diese Studie hebt hervor, wie oft die AfD-Rhetorik auf negativ wertende Adjektive zurückgreift und Gradadverbien aus dem absoluten, extrem hohen und sehr hohem Intensivierungsbereich (z. B. enorm, immens, gewaltig, äußerst, unheimlich, unendlich, usw.) einsetzt. Generell können viele skandalisierende Begriffe, die auf Konflikte verweisen, ausgemacht werden. Diese Analyse hat weiter gezeigt, dass bei der AfD diese Indikatoren im Schnitt deutlich häufiger auftreten als bei anderen Parteien (cf. Scharloth 2016). Dieser Ansicht ist auch Peter Schlobinski, der aber, obgleich er eine direkte Übereinstimmung mit der Nazi-Sprache sieht, vor Generalisierungen warnt:

Man darf nicht die gesamte AfD über einen Kamm scheren, doch einzelne Mitglieder pflegen eine auffällige Nazi-Rhetorik. Der Rhythmus, das sprachliche Diktum, die Emotionalisierung es gibt einiges, was stark an die NSDAP-Sprache angelehnt ist. Und Höcke ist ja schon fanatisch in seiner Sprache.

(Schlobinski in Ivits 2015)

Beispiele für Björn Höckes Rhetorik sind problemlos in dem dieser Studie zugrundeliegenden Analysekorpus aufzufinden. Hier exemplarisch ein Transkript aus einem Vortrag, den der Thüringer AfD-Vorsitzende am 22. November 2015 am Institut für Staatspolitik in Neuroda gehalten hat. In dieser Rede stellt er Afrikas Bevölkerungswachstum in direkten Zusammenhang mit der Flüchtlingskrise und erklärt:

[Es] ist angeraten [...] die populationsökologische Brille aufzuziehen, um den Blick noch etwas zu weiten. [...] Solange wir bereit sind, diesen Bevölkerungsüberschuss aufzunehmen, wird sich am Reproduktionsverhalten der Afrikaner nichts ändern. [...] Die Evolution hat Afrika und Europa, vereinfacht gesagt, zwei unterschiedliche Reproduktionsstrategien beschert. [...] Das Auseinanderfallen der afrikanischen und der europäischen Geburtenraten wird gegenwärtig natürlich noch durch den dekadenten Zeitgeist verstärkt, der Europa fest im Griff hat. Kurz, im 21. Jahrhundert trifft der lebensbejahende afrikanische Ausbreitungstyp auf den selbstverneinenden europäischen Platzhaltertyp.

(Höcke 2015b)

Solch ein biologisch-rassistischer Ansatz, mit dem er den Bevölkerungsüberschuss Afrikas erklärt, lässt manchen Historiker erschaudern und ruft Schriften von Joseph Arthur Graf Gobineau, etwa seinen Essai sur l'inéglité des races humaines (1853) oder Houston Stewart Chamberlains Grundlagen des 19. Jahrhunderts (1899) ins Gedächtnis, auf die sich die Ideologen der arischen Rasse beriefen. Nach der Machtergreifung der NSDAP konnte man fast täglich in der Presse solche Argumente lesen, wie aus folgendem Auszug eines Zeitungsartikels vom 10. Juni 1933 eindeutig hervorgeht: 
Die rassenhygienische und eugenische Bewegung in Deutschland kann der Regierung der nationalen Erhebung nicht dankbar genug dafür sein, dass sie als erste deutsche Regierung biologische Politik treibt. [...] Auch die Presse kann sich nicht mehr der Aufgabe entziehen, der [sic] bodenlosen Unwissenheit weitester Volkskreise in erbbiologischen und bevölkerungspolitischen Fragen zu steuern und den Grund, vererbungswissenschaftliche Kenntnisse vorzubereiten, auf dem allein rassenhygienische Einsicht und Erkenntnis reifen kann. Die jüngere Generation [...] ist ungewöhnlich dankbar für Belehrungen über die biologischen Gesetze, die das Schicksal des Einzelnen, der Familie und des Gesamtvolkes bestimmt. Sie ist bereit und gewillt, der das deutsche Volk drohenden Gefahr des Aussterbens, der Vergreisung und der Entartung entgegenzutreten.

(Konrad Dürre in: Wulf 1966: 222)

Björn Höckes sprachlicher Duktus scheint tatsächlich nicht weit entfernt von der Sprache der NSDAP zu sein, was auch Aussagen wie „Erfurt ist schön, Erfurt ist schön deutsch, und schön deutsch soll Erfurt bleiben!“ oder „3000 Jahre Europa! 1000 Jahre Deutschland“ (Höcke 2015a) unterstreichen.

Weitere erleuchtende Beispiele für den Zugriff auf Tabubruch und Skandalisierung liefert des Öfteren auch Markus Frohnmaier, einer der beiden Bundesvorsitzenden der Jungen Alternative:

Die Altparteien haben ganze Arbeit geleistet, hier im Land, im Bund, in Europa. Sie haben eine gesellschaftliche Atmosphäre der Denunziation und der Diffamierung eingerichtet. Sie haben den Rechtsbruch zum Normalfall erhoben. [...] Der Schuldige sitzt im Parlament, in der Schreibstube und im Rundfunkrat. [...] Liebe Freunde, ich sage diesen linken GesinnungsTerroristen, diesem Parteienfilz ganz klar: Wenn wir kommen, dann wird aufgeräumt, dann wird ausgemistet, dann wird wieder Politik für das Volk und zwar nur für das Volk gemacht, denn wir sind das Volk, liebe Freunde!

(Frohnmaier 2015)

Der Jura-Student Frohnmaier, selbst kein gebürtiger Deutscher, da er in Rumänien geboren und als Kind von einer deutschen Familie adoptiert wurde, ist schon oft wegen seiner Äußerungen ins Visier der Presse genommen worden. Eine Berichterstattung des ZDF-Magazins Frontal 21 und der Badischen Zeitung erhärten z. B. den Verdacht, dass Frohnmaier in der German Defence League aktiv gewesen sei, einer gewaltbereiten Organisation aus der extrem rechten Hooligan-Szene, was er natürlich im perfektem AfD-Stil dementiert (cf. Kaiser 2016).

Frohnmaiers Maxime, man solle Politik machen, „wie die ,Bild“-Zeitung schreibt, einfach, direkt, für die Menschen“" (Fiedler 2016), scheint den erfolgreichen Wahlkampf der AfD bei den Landtagswahlen 2016 in Baden-Württemberg mitbestimmt zu haben.

Besonders die neue politische Generation der Repräsentanten der Jungen Alternative spart nicht an Skandalisierungen und Tabubrüchen. Sie bezeichnen sich auch selbst gerne als die „freche Version der AfD“ (Fiedler 2016), was ihre despektierlichen Reden oft belegen. Als exemplarisch mag folgender Auszug aus der transkribierten Rede des zweiten Bundesvorsitzenden der Jungen Alternative, Sven Werner Tritschler, gelten: 
Unsere Kanzlerin hat in der Welt offenbar nicht nur den Eindruck erweckt, wir seien so eine Art Sozialstation für jeden, dem es zu Hause nicht ganz so gut geht, offenbar hat sie auch noch den Eindruck erweckt, unser Land sei ein einziges großes Freiluftbordell, in dem sich jeder mal austoben darf. Wenn Frau Merkel ein solches Etablissement leiten möchte, und das Talent will ich ihr gar nicht absprechen, dann muss sie als Kanzlerin zurücktreten, denn wir werden unsere Frauen nicht zu Freiwild machen.

(Tritschler 2016)

Diese Beispiele aus unserem Korpus sowie die schon einleitend zitierten Äußerungen von Frauke Petry und Beatrix von Storch veranschaulichen, wie in der Tat die AfD in ihrer politischen Propaganda nicht an Skandalisierungen und Tabubrüchen spart.

\subsection{Rekurs auf das Volk}

Besonders beliebt scheint in der AfD-Rhetorik der Rekurs auf die Volks-Thematik zu sein, womit sie einen Begriff aufgreift, der schon die Reden der NSDAP charakterisierte. In Cornelia Schmitz-Bernings (1998) Vokabular des Nationalsozialismus steht folgende Definition von „Volk“, in der ganz klar zu erkennen ist, dass nicht alle Angehörigen des Rechtsstaats unter diesen Begriff fallen:

Eine durch Rasse und gemeinsamen Volksboden geprägte naturhafte Gemeinschaft von gemeinsamer Abstammung, Geschichte, Sprache und Kultur, die einer starken Führung und steter Erziehung und Ausrichtung ${ }^{10}$ bedarf.

(Schmitz-Berning 1998: 642)

Mit einer Bedeutung, die dieser Definition sehr nahe kommt, charakterisiert der Begriff „Volk“ auch die Reden vieler AfD-Politiker. Das wird schon in den eben zitierten Worten Frohnmaiers deutlich, der mit der programmatischen Ankündigung abschließt: „Wenn wir kommen, dann wird aufgeräumt, dann wird ausgemistet, dann wird wieder Politik für das Volk und nur für das Volk gemacht - denn wir sind das Volk“ (Fronhmeier 2015). Ähnliches lässt sich auch bei Gauland und Petry ausmachen:

Es wird Zeit, dass wir das Schicksal des deutschen Volkes, damit es ein deutsches Volk bleibt, aus den Händen dieser Bundeskanzlerin nehmen.

(Alexander Gauland ${ }^{11}$, zitiert nach Ivits 2015)

Die deutsche Politik hat eine Eigenverantwortung, das Überleben des eigenen Volkes, der eigenen Nation sicherzustellen.

(Frauke Petry, zitiert nach Ivits 2015)

Liest man diese Zitate, erweisen sich Klemperers Sprachbeobachtungen, die er während seines ,inneren“ Exils in den Jahren des Nazi-Regimes aufzeichnete, von extremer Aktualität. Aus einem Tagebucheintrag des 20. April 1933 zitierend, berichtet er in LTI:

\footnotetext{
10 Ausrichtung: Nationalsozialistische Indoktrination (Schmitz-Berning 1998: 81); es handelt sich um eines der Worte, das im Büchlein Aus dem Wörterbuch des Unmenschen aufgenommen und beschrieben wird (Sternberg/Storz/Süskind 1962: 17-19).

11 Alexander Gauland ist Landesvoristzender der AfD in Brandenburg.
} 
«Volk» wird jetzt beim Reden und Schreiben so oft verwandt wie Salz beim Essen, an alles gibt man eine Prise Volk: Volksfest, Volksgenosse, Volksgemeinschaft, volksnah, volksfremd, volksentstammt...

(Klemperer 1996: 43)

Ähnlich wie in der NS-Zeit hebt die AfD die Zentralität des Volkswillens hervor. Die Analyse unseres Korpus belegt, dass in den Wahlkampfreden und -Programmen überdurchschnittlich häufig Okkurrenzen von Volk und dazugehörenden Komposita vorzufinden sind (AfD-Korpus 1,55\%, Vergleichskorpus 0,32\%o). Neben Volk/Völker konnten zahlreiche weitere Lexeme erhoben werden:

Volksabstimmung, Volksantrag, Volksbefragungen, Volksbegehren, Volksbeteiligung, Volksentscheid, volkserzieherisch, Volksfeste, Völkergewohnheitsrecht, Volksgruppe, Volksherrschaft, Volkshochschule, Volksinitiative, Volkspartei, Volkspetition, Völkerrecht (völkerrechtlich), Volksseuchen, Volkssouveränität, Volksvermögen, Volksverräter, Volksvertreter, Volkstod, Völkerwanderung (völkerwanderungsähnlich), Volkswirtschaft (volkswirtschaftlich), Staatsvolk.

(AfD-Korpus)

Wie man aus diesen Komposita mit dem lexikalischen Morphem \{volk\} ersehen kann, findet man neben unauffälligen Lexemen wie Volkswirtschaft oder Volkshochschule viele andere, die dem Wortfeld ,direkte Demokratie‘ zugehören (Volksabstimmung, Volksantrag, Volksbefragung, Volksbegehren, Volksbeteiligung, Volksentscheid, usw.). Von besonderem Interesse sind aber Lexeme wie Völkerwanderung, volkserzieherisch, Volkstod oder Volksseuchen, die einleuchtend die rechtspopulistische Ideologisierung des AfD-Vokabulars zum Vorschein bringen.

Wenn die AfD von Volk spricht, meint sie aber, wie schon erwähnt, nicht all diejenigen, die die deutsche Staatsbürgerschaft besitzen, unter die ja nunmehr auch viele Menschen mit Migrationshintergrund fallen. Dies wird deutlich erkennbar, wenn man die Konkordanzen des Lexems Volk untersucht. Wie folgende Screenshots der Sketch-Engine-Suchergebnisse zeigen, findet man links davon sehr oft die Lexeme deutsch*, eigen* und unser*12/dies*.

\begin{tabular}{|c|}
\hline Existenznot bringen wird. Das deutsche Volk soll deshalb nach dem britischen Vorbild \\
ihrer Kanzlerin halten wir das deutsche Volk für ebenso mündig wie das der Schweizer \\
souveränen Nationalstaat des deutschen Volkes dauerhaft erhalten. Unsere Ziele werden \\
ich meine Kraft dem Wohle des deutschen Volkes widmen, seinen Nutzen mehren, Schaden von \\
hierdurch der Riegel vorgeschoben. Das deutsche Volk ist ebenso mündig wie das der Schweizer \\
nationalen Interessen und das Wohl des deutschen Volkes im Mittelpunkt stehen. Als eine der großen \\
wir Deutschen müssen. Wir, das deutsche Volk , sind genauso wenig moralisch verpflichtet \\
hinter mir hört gut zu, wir, das deutsche Volk , sind nicht verpflichtet, unsere materielle \\
abzutreten. Aber.... Aber wir, das deutsche Volk , haben nicht nur das Recht, nein, wir haben \\
: nämlich sich "dem Wohle des deutschen Volkes " zu widmen und "Schaden von ihm (zu) wenden \\
gegen die Interessen des eigenen deutschen Volkes ! Unsere Rechtsordnung lebt wie jede andere
\end{tabular}

12 Zum exkludierenden Einsatz von unser cf. auch Kämper 2017: 13f. 


\begin{tabular}{|c|c|c|}
\hline \multicolumn{3}{|c|}{$\begin{array}{l}\text { endlich eine Politik im Interesse des eigenen Volkes gemacht wird und nicht gegen die Interessen } \\
\text { nicht gegen die Interessen des eigenen Volkes. Ich danke euch liebe Freunde. Meine sehr }\end{array}$} \\
\hline \multicolumn{3}{|c|}{ gerettet und das gegen den Willen des eigenen Volkes. Und auch bei der Asylpolitik werden es } \\
\hline \multicolumn{3}{|c|}{ müssen. Das ist Politik gegen das eigene Volk , Frau Merkel, und nach 1989 wollten wir } \\
\hline \multicolumn{3}{|c|}{ doch nie wieder Politik gegen das eigene Volk erleben. Meine Damen und Herrn... Meine } \\
\hline \multicolumn{3}{|c|}{ brauchen, die diese Verräter am eigenen Volk aus den Regierungen fegen. Liebe Freunde } \\
\hline \multicolumn{3}{|c|}{ nicht nur alle Verantwortung für das eigene Volk abgelegt, sie handeln in zynischer Weise } \\
\hline \multirow{4}{*}{\multicolumn{3}{|c|}{$\begin{array}{r}\text { Verpflichtungen gegenüber der Zukunft des eigenen Volkes sind ihr unbekannt. Sie lockt damit hunderte } \\
\text { zudem gegen die Interessen des eigenen Volkes. Unsere Rechtsordnung lebt wie jede andere } \\
\text { Linie an den Bedürfnissen unseres eigenen Volkes orientiert. Gezielte Familienförderung, } \\
\text { gegen die Interessen des eigenen deutschen Volkes ! Unsere Rechtsordnung lebt wie jede andere }\end{array}$}} \\
\hline & & \\
\hline & & \\
\hline & & \\
\hline Kirchenfürsten. Es ist der Dom unseres & Volkes & Das ist unser Dom! Und \\
\hline die Zukunft unseres Landes und unseres & Volkes & davon \\
\hline Revolution, die die Geschichte unseres & Volkes & jectu \\
\hline anzlerin", die den Eid geschworen hat, diesem & Volk & ine Nutz \\
\hline sagt und der ja zu einer Zukunft unseres & Volkes & und unserer Kinder sagt. Und i \\
\hline nenne ich eine Politik zum Schaden unseres & Volkes & \\
\hline geweckt. Der Sc & Volk & verändert. Ich denke, der Dornröschenschlaf \\
\hline
\end{tabular}

Abb. 1: Screenshots der Sketch-Engine-Suchergebnisse

Die AfD konstruiert das „Volk“ in Abgrenzung zu Asylsuchenden, Migranten und Gastarbeitern. Um es mit Worten Schlobinskis zu formulieren:

Die Idee eines deutschen Volkes, das vor der Flut beschützt werden muss, die von außen auf Deutschland zurollt, konstituiert ein Wir-Ihr-Schema. Der Volksbegriff erzeugt bei den einen ein Zugehörigkeitsgefühl und grenzt gleichzeitig andere aus dieser Volksgemeinschaft aus. Überfremdung, Asylantenflut sind beliebte Begriffe, die das Feindbild der Fremden verfestigen. Solche Wörter werden zu Kampfbegriffen, um bestimmte Menschen abzuwerten, um Grenzen zwischen ihnen und sich selbst zu ziehen. Die Fremden sind es, die das deutsche Volk bedrohen.

(Schlobinski, zitiert nach Ivits 2015)

Das Thema der Bedrohung der Bevölkerung durch Asylsuchende und Migranten hat nach den Ereignissen der Silvesternacht $2015^{13}$ noch stärkere Relevanz angenommen. Dementsprechend ist es zum Gegenstand vieler öffentlicher Äußerungen der AfD geworden.

In der populistischen Rhetorik wird diese gesellschaftliche Bedrohung mit Metaphern-Tokens und Frames wie Krankheit, Flut und Dammbruch dargestellt (cf. u. a. Tereick 2017: 409; Böke 1997: 168), die auch in unserem Korpus zahlreiche Belege finden. Besonders beliebte Themen, auf die oft gegriffen wird, sind nicht nur das unkontrollierte Einwandern von Terroristen, Verbrechern und Vergewaltigern, sondern auch die Verbreitung von Krankheiten:

Im Jaener Sozialausschuss referierte eine Vertreterin des deutschen Gesundheitsamtes [...] Krankheiten, denen man nicht vorbeugen könne, sind wieder auf dem Vormarsch. Und die Deutschen seien wegen der vermeintlichen Ausrottung von Masern, Mumps und Röteln in einem milden Impfstatus, der zu Sorge Anlass gebe. Die Bevölkerung ist nicht mehr geschützt. Volksseuchen wie die Tuberkulose, Typhus, HIV sind wieder auf dem Vormarschieren.

\footnotetext{
13 In jener Nacht kam es in Köln zu zahlreichen sexuellen Übergriffen auf Frauen durch Gruppen junger Männer, die vornehmlich aus dem nordafrikanischen und arabischen Raum stammten. In vielen Fällen wurden sowohl Sexualdelikte als auch Eigentums- und Körperverletzungsdelikte verübt.
} 
(Höcke 2015)

Die Hetze gegen diese Volksfeinde dient dazu, in der Gesellschaft eine mehr oder weniger irrationale Angst auszulösen, was laut Noam Chomsky ein sehr wirksames Instrument der Propaganda ist:

You've got to keep them pretty scared, because unless they're properly scared and frightened of all kinds of devils that are going to destroy them from outside or inside or somewhere, they may start to think, which is very dangerous, because they're not competent to think. Therefore it's important to distract them.

(Chomsky 2002: 28)

Abschließend zum Rekurs auf das Volk: Im September 2016 sorgte eine Pressemitteilung für erhebliche Aufruhr. Nach Auffassung von Frauke Petry sei es nun an der Zeit, den Begriff völkisch ohne den negativen Beigeschmack zu verwenden, der ihm in der zweiten Hälfte des 20. Jahrhunderts assoziiert wurde. Man solle ihn ja wieder positiv besetzen, sagte sie in einem Interview, denn den Begriff „,völkisch“ als rassistisch abzustempeln sei eine unzulässige Verkürzung (cf. Kamann/Balzli 2016).

Es bleibt offen, ob diese Aussage eine abermals willentliche Provokation oder ob sie ernst gemeint war und vor allem, zu welchem Zweck die Politikerin ein historisch schwer vorbelastetes Wort reinwaschen wollte. Eins steht nämlich fest und könnte jederzeit durch eine einfache Google NGram Viewer-Suche ${ }^{14}$ bestätigt werden: Die Häufigkeit des Vorkommens des Wortes völkisch weist eine enge Verbindung mit dem Naziregime auf, und zwar von Beginn an, wie in der weiteren Folge auch graphisch gezeigt wird. Schließlich hieß die Parteizeitung seit den Zwanzigern und bis zum April 1945 „Völkischer Beobachter“, und Hitler selbst nannte seine nationalsozialistische Doktrin eine „völkische“ Weltanschauung, wie man in Mein Kampf nachlesen kann:

Demgegenüber erkennt die völkische Weltanschauung die Bedeutung der Menschheit in deren rassischen Urelementen. Sie sieht im Staat prinzipiell nur ein Mittel [zur] [...] Erhaltung des rassischen Daseins der Menschen [...]. Sie glaubt somit keineswegs an eine Gleichheit der Rassen, sondern erkennt mit ihrer Verschiedenheit auch ihren höheren und minderen Wert und fühlt sich durch diese Erkenntnis verpflichtet, gemäß dem eigenen Wollen, Unterordnung des Schlechteren und Schwächeren zu verlangen. [...] Alle Gesetze und Einrichtungen des völkischen Staates dürfen nur dem Zweck dienen: Die Rasse, das Volkstum zu erhalten und zu fördern. So wird künftighin der Staat darüber wachen, daß krankes und minderwertiges Erbgut nicht willkürlich vermehrt wird.

(Hitler, Mein Kampf - zit. nach Schmitz-Berning 1998: 647)

\footnotetext{
${ }^{14}$ Durch das online Programm Google Ngram Viewer (https://books.google.com/ngrams [24.06.2019]) kann erhoben werden, wie häufig ein Wort oder eine Wortfolge im Google-Books-Textkorpus vorkommen. Das Korpus umfasst ca. 5,2 Millionen Bücher in den Sprachen Deutsch, Englisch, Französisch, Hebräisch, Russisch und Spanisch, die in den Jahren 1500-2008 gedruckt wurden. Der Benutzer kann Worte nach Belieben eingeben und ihre Gebrauchsfrequenz miteinander vergleichen. Die Darstellung erfolgt visuell in Graphen (für weitere Informationen, cf. http://litre.uni-goettingen.de/index.php/Google_Ngram_Viewer [24.06.2019]).
} 


\subsection{Frontstellung gegen die gesellschaftlichen Eliten}

Die Ablehnung des Establishments hat in der AfD zahlreiche Facetten: Sie verbreitet das Credo, dass Politik, Parteien, Medien und akademische Eliten einen Verblendungs- und Manipulationszusammenhang konstruiert hätten. In ihren Mittleilungen zeigt sich nämlich eine gewisse Neigung zu Verschwörungstheorien. Oft ist die Rede von vermeintlichen Manipulationen, Lügen oder verborgenen Wahrheiten. Fast leitmotivartig bezeichnet die AfD die Presse als „Lügenpresse“ oder „Lückenpresse“. Auch in diesem Fall wird der AfD und den anderen rechtspopulistischen Parteien vorgeworfen, sie würden NSDAP-Jargon wieder salonfähig machen wollen (cf. u. a. Ivits 2015, Neurer 2016). In der bisherigen Bestandsaufnahme konnten allerdings noch keine direkten Belege gefunden werden, die dies untermauern würden. In den dieser Studie zu Verfügung stehenden sprachlichen Dokumenten des Nazi-Regimes kommt der Begriff „Lügen“- bzw. „Lückenpresse“ nicht vor (cf. Wulf 1966), und ebenso wenig erfolgreich war die Suche danach in der digitalen Gesamtausgabe von Victor Klemperers Tagebüchern

(cf. Nowojski 2007). Dass der Philologe, der bekanntlich mit pedantischer Genauigkeit die Sprache des NSDAP-Regimes unter die Lupe nahm, keine Angaben darüber macht, mag ein Indiz dafür sein, dass solche Begriffe nicht unbedingt als konnotativ für die Nazisprache gelten können. Eine Bestätigung dafür findet sich ebenfalls durch eine Worthäufigkeitsanalyse mit Google NGram Viewer. Gibt man hier das Wort Lügenpresse ein, ergibt sich folgendes Diagramm.



Grafik 1: Lügenpresse im deutschen Google-Buchbestand im Zeitraum 1900-2008.

Aus dieser graphischen Darstellung wird ersichtlich, dass der Begriff schon vor dem NSDAPRegime im sprachlichen Usus war, und zwar mit einer höheren Okkurrenz während des Ersten Weltkrieges. Auffällig ist auch, dass die Prozentzahlen der Okkurrenzen extrem niedrig sind, vor allem wenn man sie einem hoch konnotierten Begriff wie etwa dem schon besprochenen ,völkisch“ gegenüberstellt. 


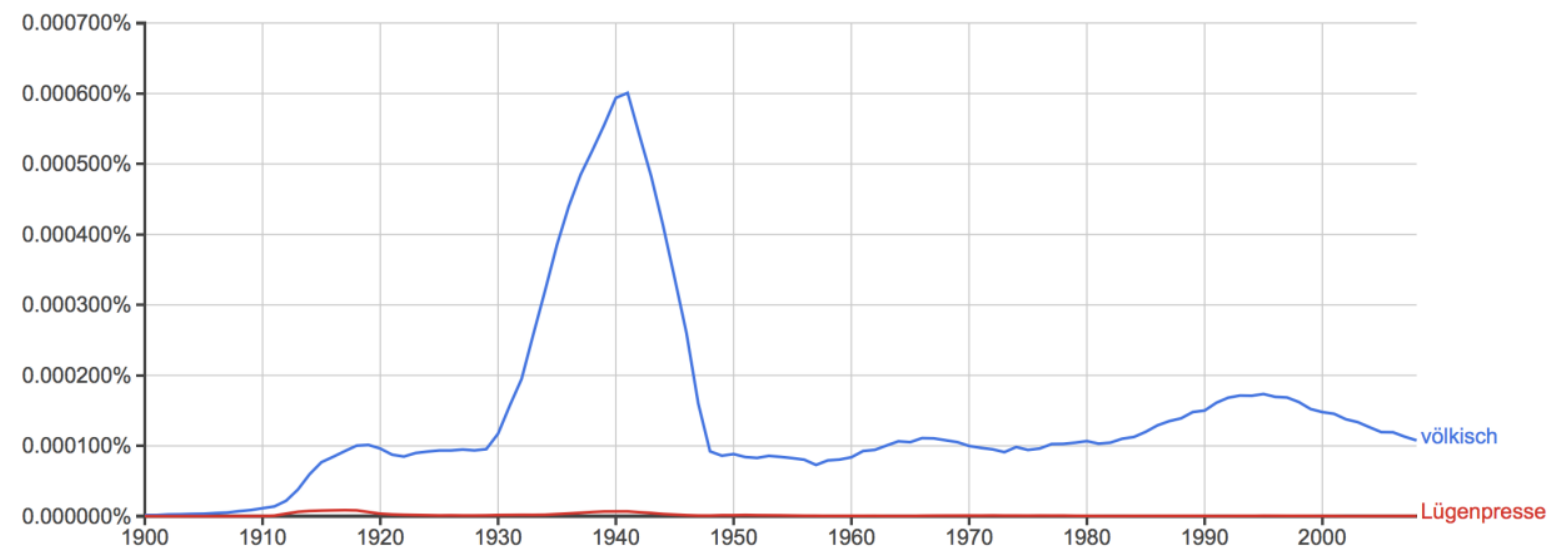

Grafik 2: Lügenpresse vs. völkisch im deutschen Google-Buchbestand im Zeitraum 1900-2008.

An diesem Diagramm lässt sich die Konnotation des Begriffs völkisch ablesen, da die Kurve zeigt, dass der Einsatz des Wortes in einer ganz bestimmten Zeitspanne vorkommt. Im Falle von Lügenpresse benutzt hingegen die AfD ein Wort, das nicht direkt mit der Idiomatik des Nationalsozialismus verbunden werden kann. Goebbels' Propaganda richtete sich zwar auch stark gegen die Presse, aber vornehmlich sprach sie von „Schmutzpresse“, „Juden-Presse“, „Bolschewistische Presse“ und vor allem von „Systempresse“. In der Tat kann man in Grafik 3 sehen, dass Systempresse nur in den Jahren des Nationalsozialismus Okkurrenzen aufweist.

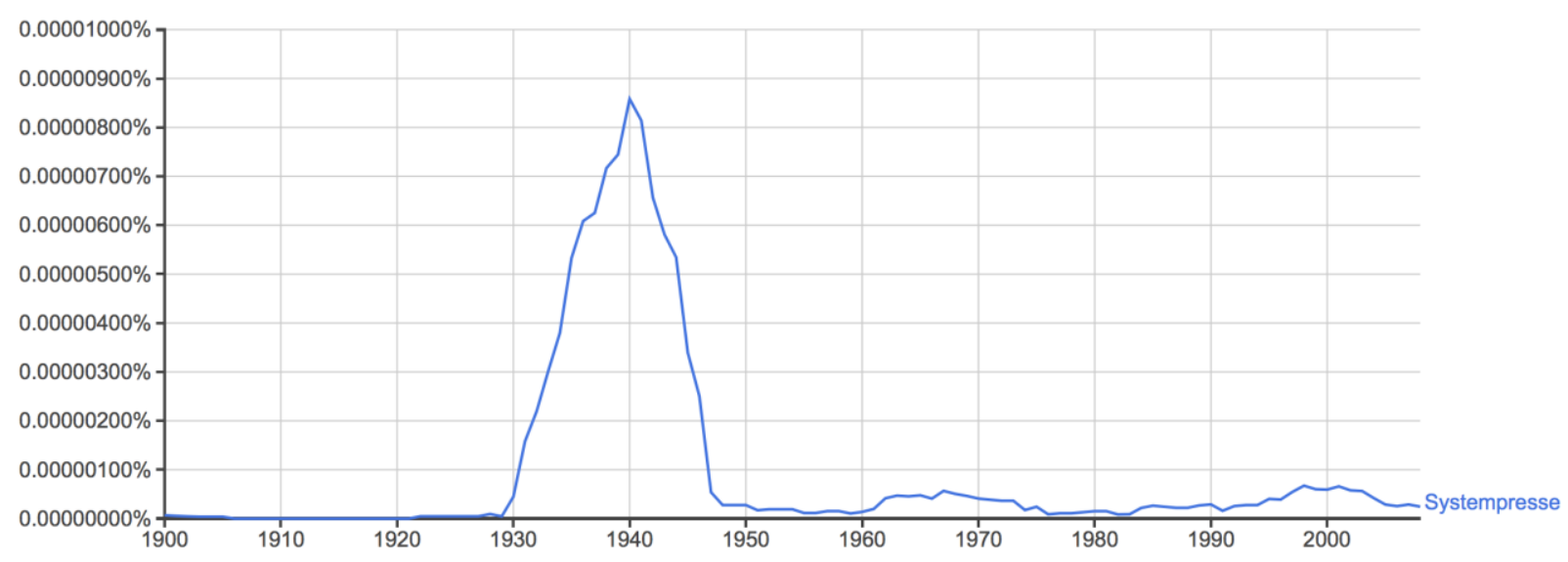

Grafik 3: Systempresse im deutschen Google-Buchbestand im Zeitraum 1900-2008.

Mit „System“ bezeichnete das NSDAP-Regime all das, was mit der Weimarer Republik zu tun hatte. Als korrupte Vertreter des Systems wurden die Gegnerparteien genannt. Und die Gegenparteien sind wiederum ein Punkt, in dem die AfD ihre Frontstellung gegen die gesellschaftlichen Eliten zeigt. Politische Konkurrenten bezeichnet sie mit Vorliebe als „Systemparteien“, oder gar als „Altparteien“; es ist oft auch von einer verschwörungstheoretischen „Parteienherrschaft" die Rede. So liest man in der Präambel des Grundsatzprogramms: 
Heimlicher Souverän ist eine kleine, machtvolle politische Führungsgruppe innerhalb der Parteien. Sie hat die Fehlentwicklungen der letzten Jahrzehnte zu verantworten. Es hat sich eine politische Klasse von Berufspolitikern herausgebildet, deren vordringliches Interesse ihrer Macht, ihrem Status und ihrem materiellen Wohlergehen gilt. Es handelt sich um ein politisches Kartell, das die Schalthebel der staatlichen Macht, soweit diese nicht an die EU übertragen worden ist, die gesamte politische Bildung und große Teile der Versorgung der Bevölkerung mit politischen Informationen in Händen hat.

(AfD 2016: 8)

Die AfD selbst hingegen trete der allgegenwärtigen Manipulation mit den Mitteln der Wahrheit und der Vernunft entgegen. Sie verwendet zu ihrer Selbstkonzeption stereotypisierende Elemente, die sie mit den Eigenschaften „demokratisch“, „sachlich“ und „,verlässlich“ versieht (cf. Kämper 2017: 4) und bezeichnet sich selbst als:

Volkspartei, Rechtsstaatspartei, Weckruf-Partei, Konfliktpartei, Mitmachpartei, AntiEuropartei, Oppositionspartei, Partei des gesunden Menschenverstands, Aufräumer-Partei.

(AfD-Korpus)

Hier kann man eine inhaltliche Übereinstimmung mit der NSDAP-Rhetorik ausmachen, die bekanntlich das Parteiensystem der Weimarer Republik pauschal als überkommene Institution abwertete.

Aufschlussreich ist auch die Analyse markiert gebrauchter Ausdrücke: Setzt man Wörter in Anführungszeichen oder distanziert man sich von einer Bezeichnung, indem man ein sogenannt davorsetzt, ist dies ein Indikator für Kritik. Scharloth (2016) hebt hervor, dass sich die AfD weit häufiger als andere Parteien des demokratischen Spektrums metasprachlicher Markierungen bedienen. Hier einige Beispiele aus unsere Korpus:

Steuerverschwendung durch „Gender-Forschung“ beenden: Die „Gender-Forschung“ ist keine seriöse Wissenschaft [...]. Bund und Länder dürfen keine Mittel für die „Gender-Forschung“ mehr bereitstellen und keine „Gender-Professuren“ mehr besetzen. Bestehende Förderlinien sollen beendet werden, die der „Gender-Ideologie“ verpflichteten „Gleichstellungsbeauftragten“ an den Universitäten sind abzuschaffen.

(AfD 2017: 36)

Im Inland sieht die AfD mit Sorge, wie die deutsche Sprache im Sinne einer falsch verstandenen „Internationalisierung“ durch das Englische ersetzt oder „gegendert“ wird. Politisch „korrekte“ Sprachvorgaben lehnen wir entschieden ab.

(AfD 2016: 47)

Der Rechtsstaat muss für alle Bürger gleichermaßen gelten. Es darf keinen „kulturellen Rabatt“ für bestimmte Straftaten, wie z. B. strafmildernde Umstände bei Ehrenmord, geben. Der Aufbau paralleler Rechtsstrukturen durch sogenannte „Friedensrichter“ muss konsequent unterbunden werden, da sie den Rechtsstaat aushebeln.

(AfD Berlin 2016: 17)

Und wieder finden wir in Klemperers Worten ein direktes Pendant hierzu:

Man sollte annehmen, daß die LTI, da sie doch im Kern rhetorisch ist und sich immer wieder an das Gefühle wendet, ähnlich wie der Sturm und Drang dem Ausrufezeichen ergeben sein müßte. Das ist kaum auffällig; im Gegenteil, sie scheint mir ziemlich sparsam mit diesem Zeichen umzugehen. Es ist, als forme sie alles mit solcher Selbstverständlichkeit zu Anruf und Ausruf, daß 
sie dafür gar kein besonderes Interpunktionszeichen nötig habe - denn wo sind die schlichten Aussagen, von denen sich der Ausruf abheben müßte? Dagegen bedient sich die LTI bis zum Überdruß dessen, was ich die ironischen Anführungszeichen nennen möchte.

(Klemperer 1996: 94)

Ironische Anführungszeichen werden nicht nur in der Schriftsprache benutzt, sondern finden ihren Einsatz auch in der gesprochenen Rhetorik. Hier einige Beispiele aus Björn Höckes Reden. Seine ironischen Anführungszeichen entsprechen meistens gezielt eingesetzten Sprechpausen, denen eine skandierte und langsame Aussprache des betroffenen Ausdrucks folgt. Des Weiteren greift er auf Minenspiel oder auf den erläuternden Einsatz des Idiomatischen „in Anführungszeichen“ zurück:

Der größte Sandmann, liebe Freunde, der größte Sandmann kommt aus Bayern und er heißt: Horst Seehofer. Dieser [Sprechpause und dann langsam ausgesprochen] Politikfunktionär [Sprechpause und ironischer Gesichtsausdruck], der ist dort, wo es um das Durchsetzen seiner Machtoption geht, wahrlich ein Willensriese, aber dort, wo es um die Durchsetzung deutscher Interessen auch gegen den Zeitgeist geht, da ist er ein Willenszwerg. [...] Herr [Sprechpause] Ministerpräsident [Sprechpause] Horst Seehofer, ich appelliere hier und heute Abend aus Erfurt noch mal an Sie: Versuchen Sie doch ihren Funktionärsgeist zu überwinden.

$[\ldots]$

Unter Angela Merkel, unsere in Anführungszeichen „Bundeskanzlerin“, die den Eid geschworen hat, diesem Volk zu dienen, seine Nutzen zu mehren und Schaden von ihm abzuwenden, ist diese Republik zu einer Bananenrepublik verkommen.

(Höcke 2016a)

\section{$3 \quad$ Fazit}

Wollte man aus dieser Bestandsaufnahme ein Fazit ziehen, so schiene es doch so zu sein, dass in den neuen Schläuchen der rechtspopulistischen Parteien (zur Zeit) recht alter Wein fließe. Dieser Versuch einer primär lexikalisch orientierten Untersuchung zeigt nämlich, dass sich nicht wenige Analogien zwischen der nationalsozialistischen Sprache und den Wahlkampfreden der AfD erkennen lassen.

Weitere Analysen des dieser Studie zugrundeliegenden Analysekorpus würden den Rahmen dieses Beitrags sprengen, doch es ließen sich zweifelsohne noch weitere interessante Aspekte erforschen. Auf lexikalischer Ebene könnte z. B. das Phänomen der Komplexitätsreduzierung der politischen Sprache und die ideologische Polysemie des Wortguts fokussiert werden, während auf syntaktischer Ebene die Prädominanz des parataktischen, linearen Satzbaus im Gegensatz zu dem meist hypotaktischen Stil der spekulativen Rhetorik der eher konservativen Parteien zu verifizieren wäre.

In Anbetracht der Wahlergebnisse des letzten Jahres sind solche Studien im heutigen weltweiten politischen Panorama von höchstem Interesse, denn viel zu oft wird Sprache als Mittel der Persuasion und Machtausübung unterschätzt. Dolf Sternberger schreibt im Vorwort zur ersten Ausgabe seines Aus dem Wörterbuch des Unmenschen:

Sprache ist die Gabe allein des Menschen, das verwirrende und befreiende, verräterische und erhellende, ausgreifende und fesselnde, lösende und bindende, selige und gefährliche Medium und Siegel seines Wesens. Soviel und welche Sprache einer spricht, soviel und solche Sache, Welt oder Natur ist ihm erschlossen. Und jedes Wort, das er redet, wandelt die Welt, worin er 
sich bewegt, wandelt ihn selbst und seinen Ort in dieser Welt. Darum ist nichts gleichgültig an der Sprache, und nichts so wesentlich wie die façon de parler. Der Verderb der Sprache ist der Verderb des Menschen. Seien wir auf der Hut! Worte und Sätze können ebensowohl Gärten wie Kerker sein, in die wir, redend, uns selbst einsperren, und die Bestimmung, Sprache sei allein die Gabe des Menschen oder eine menschliche Gabe, bietet keine Sicherheit. Denn der Begriff des Menschen schließt die Möglichkeit (und Wirklichkeit) des Unmenschen in sich.

(Sternberger/Storz/Süskind 1962: 9)

\section{Literaturverzeichnis}

Böke, Karin (1997): „Die ,Invasion’ aus den ,Armenhäusern Europas’. Metaphern im Einwanderungsdiskurs“. In: Jung, Matthias/Wengler, Martin/Böke, Karin (eds.): Die Sprache des Migrationsdiskurses. Opladen, Westdeutscher Verlag: 164-193.

Carofiglio, Gianrico (2010): La manomissione delle parole. Milano: Rizzoli.

Cedroni, Lorella (2014): Politolinguistica. L'analisi del discorso politico. Roma: Carocci.

Chomsky, Noam (2015): Propaganda and the Public Mind. London: Pluto Press.

Chomsky, Noam (2006): Guerra e propaganda. La verità della guerra e la verità dei grandi media. Roma: Datanews.

Chomsky, Noam (2002): Media Control. The Spectacular Achievements of Propaganda. New York: Seven Stories Press.

Collard, Chistopher/Cropp, Martin (2008): Euripides. Fragments. London: Harvard University Press. (Loeb classical library 504).

Duden online: www.duden.de/rechtschreibung [15.05.2018].

Ehlich, Konrad (ed.) (1989): Sprache im Faschismus. Frankfurt a. M.: Suhrkamp.

Elmer, Christina/Holscher Max (2017): „CDU vor SPD, AfD verliert“. Spiegel 4.04.2017 www.spiegel.de/poli-tik/deutschland/cdu-legt-leicht-zu-afd-verliert-an-zustimmung-derspon-wahltrend-a-1141780-druck.html [19.05.2017].

Enzi, Aldo (2012): Il lessico della violenza nella Germania nazista. L'uso delle parole come strumento di propaganda, persuasione e sopraffazione nel Terzo Reich. Milano: Pgreco Edizioni.

Evans, Richard J. (2011): Il Terzo Reich al potere. 1933-1939. Milano: Mondadori.

Fahnestock, Jeanne (2011): Rhetorical Style. The Uses of Language in Persuasion. Oxford etc.: Oxford University Press.

Fiedler, Maria (2016): „Markus Frohnmaier von der Jungen Alternative: ,Wir sind die freche Version der AfD'“. Tagespiegel 16.03.2016 www.tagesspiegel.de/politik/markusfrohnmaier-von-der-jungen-alternative-wir-sind-die-freche-version-der-afd/13319676.html [25.09.2016].

Göttert, Karl-Heinz (2010): Deutsch. Biografie einer Sprache. Berlin: Ullstein.

Grebing, Helga (1961): Der Nationalsozialismus. Ursprung und Wesen. München: Olzog.

Ivits, Hellen (2015): „Hetzsprache der Rechten. So sehr verfällt die AfD in Nazi-Jargon“. Stern 30.10.2015: www.stern.de/politik/deutschland/afd-verfaellt-durch-bjoern-hoeckefrauke-petry-und-co-zunehmend-in-nazi-jargon-6524228.htm [22.09.2016].

Jacobs, Peter (2010): Victor Klemperer. Im Kern ein deutsches Gewächs. Eine Biographie. Berlin: Aufbau. 
Kaiser, Sebastian (2016): „Neue Vorwürfe gegen AfD-Jungpolitiker Frohnmaier“. Badische Zeitung 26.07.2016: www.badische-zeitung.de/suedwest-1/neue-vorwuerfe-gegen-afdjungpolitiker-frohnmaier-125381174.html [20.05.2017].

Kamann, Matthias/Balzli, Beat (2016): „Petry will den Begriff ,völkisch’ positiv besetzen“. Die Welt 11.09.2016: www.welt.de/politik/deutschland/article158049092/Petry-will-denBegriff-voelkisch-positiv-besetzen.html [26.09.2016].

Kämper, Heidrun Deborah (2017): „Das Grundsatzprogramm der AfD und seine historischen Parallelen. Eine Perspektive der Politolinguistik“. Sprachreport 2: 1-21.

Klemperer, Victor (2011): LTI. La lingua del Terzo Reich. Taccuino di un filologo. Nuova edizione riveduta e annotate a cura di Elke Fröhlich. Firenze: Giuntina.

Klemperer, Victor (1999): Tagebücher 1933-1934. Berlin: Aufbau-Verlag.

Klemperer, Victor ([1947] 1996): LTI. Notizbuch eines Philologen. Leipzig: Reclam.

Le Nouveau Petit Robert: https://pr.bvdep.com/robert.asp [15.05.2018].

Lewandowski, Marcel (2014): „Populismus in sozialen Netzwerken: Die Beispiele AfD und pro Deutschland“. Osnabrücker Beiträge zur Sprachtheorie 86: 19-46.

Matthies, Bernd (2016): „Die Entschuldigung mit der Maus. Beatrix von Storch ist ausgerutscht“. Der Tagesspiegel 09.02.2016: www.tagesspiegel.de/politik/beatrix-von-storchist-abgerutscht-die-entschuldigung-mit-der-maus/12936622.html [27.05.2017].

Neuerer, Dietmar (2016): „AfD-Jargon. CDU-Politiker fühlt sich an Nazi-Zeit erinnert“. Handelsblatt 17.04.2016: www.handelsblatt.com/politik/deutschland/afd-jargon-cdupolitiker-fuehlt-sich-an-nazi-zeit-erinnert/v_detail_tab_print/13601678.html [13.09.2016].

Nowojski, Walter (ed.) (2007): Victor Klemperer: Die Tagebücher 1933-1945. Kommentierte Gesamtausgabe. Berlin: Directmedia. (= Digitale Bibliothek 150).

Ott, Clara (2016): „Frauen sind anders als Kinder verständig“. Die Welt 01.02.2016: www.welt.de/politik/deutschland/article151705994/Frauen-sind-anders-als-Kinderverstaendig.html [27.05.2017].

Perelman, Chaim/Olbrechts-Tyteca, Lucie (1970): Traité de l'argumentation. La nouvelle rhétorique. Bruxelles: Èd. De l'Université Libre de Bruxelles.

Petrilli, Raffaella (ed.) (2015): La lingua politica. Lessico e strutture argomentative. Roma: Carocci.

Scharloth, Joachim (2016): Ist die AfD eine populistische Partei? - Eine Analyse am Beispiel des Landesverbands Rheinland-Pfalz. www.security-informatics.de/blog/?p=1790 [17.05.2017].

Schiller, Friedrich (2013): Xenien und Votivtafeln. Berlin: Edition Holzinger.

Schlosser, Horst Dieter (2013): Sprache unterm Haken-Kreuz. Eine andere Geschichte des Nationalsozialismus. Köln: Böhlau.

Stegemann, Bernd (2017): Das Gespenst des Populismus. Ein Essay zur politischen Dramaturgie. Berlin: Theater der Zeit.

Steffen, Tilman (2016): „Provozieren, relativieren, dementieren“. Die Zeit 31.05.2016: www.zeit.de/politik/deutschland/2016-05/afd-jerome-boateng-alexander-gauland-fraukepetry-medien-bjoern-hoecke [19.05.17].

Sternberger, Dolf/Storz, Gerhard/Süskind, Wilhelm E. (1962): Aus dem Wörterbuch des Unmenschen. München: DTV. 
Tereick, Jana (2017): „Risikodiskurse“. In: Thomas Niehr/Kilian, Jörg/Wengeler, Martin (eds.): Handbuch Sprache und Politik. Bremen, Hempen: 407-418. (= Sprache - PolitikGesellschaft 21.2).

Tomik, Stefan (2016): „AfD-Vizechefin will Polizei sogar auf Kinder schießen lassen“. Frankfurter Allgemeine Zeitung 16.03.2016, www.faz.net/aktuell/politik/fluechtlingskrise/beatrix-von-storch-afd-vizechefin-willpolizei-sogar-auf-kinder-schiessen-lassen-14044186.html [27.05.2017].

Wodak, Ruth (2015): The Politics of Fear. What Right-Wing Populist Discourses Mean. London: Sage.

Wulf, Joseph (1966): Presse und Funk im Dritten Reich. Eine Dokumentation. [Erscheinungsort nicht ermittelbar]: Rowohlt.

Zagrebelsky, Gustavo (2010): Sulla lingua del tempo presente. Torino: Einaudi.

Zicht, Wilko/Cantow, Matthias (2017): Wahlergebnisse. In: www.wahlrecht.de/ergebnisse/ [16.05.2017].

\section{Korpus}

AfD (2017): Programm für die Wahl zum deutschen Bundestag am 24. September 2017. Leitantrag der Bundesprogrammkommission zum Bundesparteitag am 22./23.04.2017 in Köln. In: $\quad$ www.alternativefuer.de/wp-content/uploads/sites/111/2017/03/2017-0308_afd_leitantrag-bpt-btw-programm_mit-zeilennummern-1.pdf [27.05.2017].

AfD (2016): Programm für Deutschland. Das Grundsatzprogramm der Alternative für Deutschland. $\quad$ www.afd.de/wp-content/uploads/sites/111/2017/01/2016-06-27_afdgrundsatzprogramm_web-version.pdf [27.05.2017].

AfD Baden-Württemberg (2016): Landtagswahlprogramm 2016 der AfD BadenWürttemberg. https://afd-bw.de/afdbw/wahlprogramme/landtagswahlprogramm_afd_2016_1.pdf [27.05.2017].

AfD Berlin (2016): Berlin braucht Blau. Das Wahlprogramm der AfD Berlin. https://afd. berlin/wp-content/uploads/2016/07/AfD_Berlin_Wahlprogramm_A5_RZ.pdf [27.05.2017].

AfD Rheinland-Pfalz (2016): Wahlprogramm des Landesverbades Rheinland-Pfalz der Alternative für Deutschland zur Landtagswahl 2016. http://rlp.afd-hosting.de/wpcontent/uploads/sites/110/2015/11/wahlprogramm-ausfuehrlich.pdf [27.05.2017].

AfD Sachsen (2014): Wahlprogramm $2014 \quad$ (Langfassung). www.afdsachsen.de/download/AfD_Programm_Lang.pdf [27.05.2017].

AfD Sachsen-Anhalt (2016): Wahlprogramm zur Landtagswahl am 13. März 206. www.sachsen-anhaltwaehlt.de/fileadmin/LTW2016/Wahlprogramme/wahlprogramm_afd.pdf [27.05.2017].

Bündnis90/Grünen (2013): Bündnis90/Die Grünen Bundeswahlprogramm 2013. www.gruene.de/fileadmin/user_upload/Dokumente/Wahlprogramm/Wahlprogrammbarrierefrei.pdf [30.01.2017].

CDU/CSU (2013): CDU/CSURegierungsprogramm 2013/2017. www.cdu.de/sites/default/files/media/dokumente/regierungsprogramm-2013-2017langfassung-20130911.pdf [30.01.2017].

FDP (2013): FDP Bürgerprogramm 2013. www.fdp.de/files/408/B_rgerprogramm A5_Online_2013-07-23.pdf [23.01.2017]. 
Frohnmaier, Markus (2015): Rede in Erfurt (28.10.2015). www.youtube.com/watch?v $=6 \mathrm{znCu} 1 \mathrm{VMr} 5 \mathrm{Q} \& \mathrm{t}$ [20.05.2015].

Höcke, Björn (2016a): Rede in Erfurt (13.01.2016). www.youtube.com/watch?v $=$ tqyao $910 C c Y$ [27.05.2017].

Höcke, Björn (2016b): Rede in Jena (20.01.2016). www.youtube.com/watch?v $=7 \mathrm{nfzuPz} 7 \mathrm{Rkw}$ [27.05.2017].

Höcke, Björn (2015a): AfD-Demo in Erfurt (23.09.2015). www.youtube.com/watch?v $=\mathrm{iHnjuJ} 3 \mathrm{fdwE} \& \mathrm{t}[20.05 .2017]$.

Höcke, Björn (2015b): Asyl. Eine politische Bestandsaufnahme (Rede am Institut für Staatspolitik - 22.11.2015). www.youtube.com/watch? $\mathrm{v}=\mathrm{ezTw} 3$ ORSq1Q\&t=233s [20.05.2017].

Linke (2013): DIE LINKE: Bundestagswahlprogramm 2013. https://download.dielin-

ke.de/fileadmin/download/wahlen2013/bundestagswahlprogramm/bundestagswahlprogram m 2013_langfassung.pdf [30.01.2017].

Petry, Frauke (2015): Rede an der AfD Großdemo in Berlin (7.11.2015). www.youtube.com/watch?v=tip9ljzXdXg [27.05.2017].

$\begin{array}{lllll}\text { SPD } & \text { (2013): } & \text { SPD } & \text { Regierungsprogramm } & \text { 2013/2017. }\end{array}$ www.spd.de/linkableblob/96686/data/20130415_regierungsprogramm_2013_2017.pdf [23.01.2017].

Storch von, Beatrix (2015): Rede an der AfD Großdemo in Berlin (7.11.2015). www.youtube.com/watch?v=2gAvCMlCQ_4 [27.05.2017].

Tritschler, Sven Werner (2016): Man hat gesagt, das ist Willkommenskultur, das halten wir aus. (Rede in Erfurt, 13.01.2016). www.youtube.com/watch?v=vhdX3GYuuiM [21.05.2017]. 